\title{
Prevalence of Liver Disease in Russia's Largest City: A Population-based Study
}

\author{
Komova A. , Maevskaya M., Ivashkin V. \\ Hepatology department, I.M.Sechenov First State Medical University, Moscow, Russia \\ *Corresponding author: annakomova@inbox.ru
}

Received August 16, 2014; Revised October 10, 2014; Accepted October 16, 2014

\begin{abstract}
Background and aim: Difficulties in accessing data from individual countries, especially Russia, hinder global evaluation of liver disease in Europe. The aim of this study is to estimate the prevalence of liver disease in Moscow, Russia's capital and most densely populated urban area. Methods: We recruited 5,000 random Moscow residents over the age of 18 in a population-based cross-sectional study. Participants were screened for liver disease between October 2012 and November 2012. Socio-demographic, health and lifestyle information was obtained via questionnaire; alcohol screening was done using CAGE and AUDIT tools; BMI was measured by physical exam; liver tests (ALT, AST, GGTP, bilirubin, ALP, ALT/AST), anti-HCV, HBsAg, and $\gamma$-globulins were checked by laboratory analysis; an abdominal ultrasound was performed for all patients. Provisional diagnoses were formed for all participants according to our established algorithm. Results: In total, 4,768 participants completed screening. Socio-demographic data: male 1,671 (33.42\%), female 3,329 (66.58\%), average age 45 years, average BMI $26.2 \mathrm{~kg} / \mathrm{m}^{2}$. Average BMI $>25 \mathrm{~kg} / \mathrm{m}^{2}$ was observed among the following occupational groups: retired, disabled, military and businessmen. Health care workers and students were the "slimmest" occupational groups, with only $24.7 \%$ and $21.8 \%$ having BMI $>25 \mathrm{~kg} / \mathrm{m}^{2}$ respectively. The CAGE and AUDIT questionnaires revealed that a huge number of participants (74.64\% of all participants) should reduce the quantity of alcohol they consume, and that 9.8\% abuse alcohol or are alcohol dependent. Overall, 1,459 subjects (30.6\%, m:f=2:1) had at least one abnormality in liver function tests. We found the following prevalence of liver diseases in Russia's largest city: NAFLD 7.4\% $(\mathrm{n}=352)$, ALD 6.9\% $(\mathrm{n}=329)$, hepatitis C $6.7 \%(\mathrm{n}=322)$, hepatitis B 1.9\% $(\mathrm{n}=91)$, DILI $0.82 \%(\mathrm{n}=39)$, cholestasis $0.69 \%(n=33)$, AIH $0.78 \%(n=37)$, other forms of liver disease $5.4 \%(n=258)$. Conclusions: According to our study, more than one quarter of Moscow residents have abnormal liver tests that may indicate liver disease. NAFLD, ALD and hepatitis $\mathrm{C}$ were the main causes of abnormal tests. The primary risk factors for abnormal liver tests as determined by multivariable analysis were: young age (30-59 years, $\mathrm{p}<0.001$ ), alcohol abuse (AUDIT score $>16$, $\mathrm{p}<0.04$ ), and hypercholesterolemia $(\mathrm{p}=0.016) .80 .3 \%$ of patients with abnormal liver tests required etiotropic treatment.
\end{abstract}

\section{Keywords: Russia, prevalence, liver disease, epidemiology}

Cite This Article: Komova A., Maevskaya M., and Ivashkin V., "Prevalence of Liver Disease in Russia's Largest City: A Population-based Study.” American Journal of Clinical Medicine Research, vol. 2, no. 5 (2014): 99-102. doi: 10.12691/ajcmr-2-5-2.

\section{Introduction}

Diffuse liver disease is the most significant gastroenterological illness nowadays. The rate of liver disease is increasing around the world and approximately 29 million persons in the European Union suffer from a chronic liver condition [13]. Liver disease can be silent and asymptomatic for decades and first present an advanced stage (for example, as complications of portal hypertension). Liver cirrhosis accounted for $1.8 \%$ of all deaths in Europe in 2000-2002 [13]. Alcohol abuse and non-alcoholic fatty liver disease (NAFLD) are two factors with the potential to keep levels of liver cirrhosis relatively high in European countries.

Data on the prevalence of liver disease in Russia are usually incomplete and are not included in official reports published by the World Health Organization (WHO) or other international organizations. Some groups of the Russian population - including pregnant women, blood donors, health care workers, and military personnel - are regularly screened for viral hepatitis, but there is no standard screening process for the general population.

Liver disease is becoming increasingly common among the working age populations $[2,3,7,9,13]$. We reviewed current data about liver epidemiology and found that the true prevalence of liver disease in the world is not known and that further investigation is needed.

The aim of our research was to estimate the prevalence of liver disease in Moscow, Russia's capital city and most densely populated urban area. To do so, we collected demographic data from the study population, calculated the frequency of abnormal liver tests and made preliminary diagnoses for participants with abnormal tests. 
We analyzed liver disease risk factors and selected the most important factors in order to make suggestions about liver disease prophylaxis and early detection in Russia.

\section{Methods}

Our study was a population-based cross-sectional study based in Moscow, Russia. A total of 5,000 random Moscow residents between the ages of 18 and 75 years old were enrolled (power - 90.6\%). Of these subjects, 4,768 completed all required evaluations. 232 subjects were excluded from the study because of incomplete data.

Study recruitment was organized around the social project "Test your liver", which was sponsored by the Russian Scientific Liver Society. Participants received information about the project via outdoor advertising, banners, booklets, and the websites of major Russian medical organizations (rsls.ru, gastro.ru, gastrohep.ru). The participant flow diagram for recruitment and retention is presented in Figure 1.

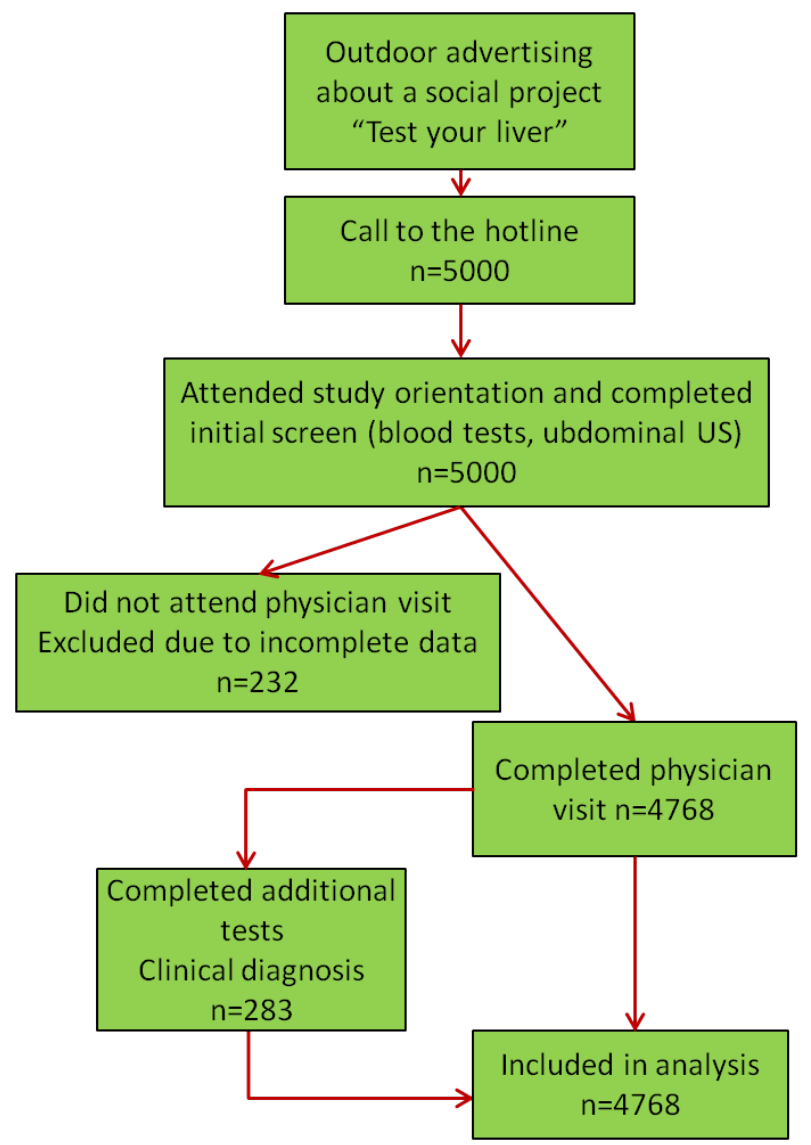

Figure 1. Participant flow of recruitment and retention for the population-based cross-sectional study

The following information was collected from each participant:

1. Demographic data including age and sex.

2. Professional occupation (laborer, office professional, retired, disabled, homemaker, student, healthcare worker, academic or cultural worker, manager/business owner, educational worker, military or other).

3. Health related behaviors: tobacco use, alcohol screening using the CAGE and Alcohol Use Disorders Identification (AUDIT) tests, dietary habits as measured by food diary.
4. Medication history: any prescription medications, herbal medicines or dietary supplements used during the last 3 months.

5. Physical examination: including height, weight and body mass index (BMI), the ratio of weight in $\mathrm{kg}$ to height in square meters.

6. Laboratory screening tests to identify common forms of liver disease: complete blood count, chemistry panel (ALT, AST, total bilirubin, direct bilirubin, GGT, AP, triglycerides, cholesterol, glucose), protein electrophoresis, anti-HCV, and HBsAg [5,8,12].

7. Abdominal ultrasound.

8. As needed: additional physical examination from a general practitioner.

The 4,768 subjects who completed the full protocol were divided into 2 groups for further analysis. Group A included participants with abnormal liver function tests and group B included participants without such changes. Univariable and multivariable statistical analysis with binary logistic regression was done to compare the groups.

\section{Results}

Our population-based sample of Moscow residents included 5,000 people. Women made up $66 \%$ of our sample $(n=3329)$ and men made up $33.4 \%$ of the sample $(n=1671)$. From this, we concluded that women were more concerned about their health and thus more likely to enroll.

Participants were broken into three age categories: young age (18-44 years) $-47.5 \%$ of sample, middle age (45-59 years) - 33.4\%, and advanced age (60-75 years) $19,1 \%$.

Further analysis of the 4,768 participants who complied all study requirements revealed 1,461 persons (30.6\% of total) with possible liver disease (study Group A). Based on this finding, we estimate that more than one in four Moscow residents have abnormal liver tests that may indicate liver disease. Group B, participants with normal liver tests, included 3,307 people (69.4\% of total). A male predominance was found among participants with abnormal liver tests: $49.7 \%$ of Group A was male, whereas only $25.5 \%$ of Group B was male $(\mathrm{p}<0.001)$. This is very interesting because the overall male to female ratio of the study population was 1:2.

A great amount of clinical research around NAFLD and alcoholic liver disease (ALD) risk factors has been done in the last several years. Based on initial diagnoses assigned in our study, the proportion of such diseases was very large: $24.1 \%$ for NAFLD and $18.1 \%$ for ALD (42.2\% total). NAFLD was directly associated with obesity and metabolic syndrome. These pathological conditions become more frequent with age, but according to recent data NAFLD is not associated with advanced age [1]. In our study we found the same results: advanced age is an independent preventive factor for abnormal liver tests.

We also analyzed sex, BMI, CAGE and AUDIT scores (used to screen for alcohol abuse), smoking, and specific laboratory measurements (cholesterol, triglycerides, glucose, GGT, gamma-globulins) as independent risk factors for liver disease.

\subsection{Sex}


Male sex was a significant risk factor for liver disease $(\mathrm{p}<0.001)$. However, male sex became insignificant during multivariable analysis $(\mathrm{p}=0.244)$ and was ultimately excluded from the logistic regression model. We can explain this with the generally known fact that male sex is an independent and significant risk factor for ALD [1,6,10,14] and therefore for alcohol abuse, and during multivariable analysis alcohol abuse (AUDIT score) in our study was singled out as a stronger factor.

\subsection{BMI}

Findings from published research show that BMI is an independent risk factor for NAFLD development $[1,7,8,11]$. In our study overweight and obesity were also identified as a significant causes of elevated liver tests. However, in multivariable analysis BMI was not found to be significant. We suppose this is connected with the fact that obesity is not widespread in Russia. The average BMI of Moscow residents is not very high: $26.5 \mathrm{~kg} / \mathrm{m}^{2}$ for males and $26.3 \mathrm{~kg} / \mathrm{m}^{2}$ for females in our study. BMI in Group A, the patients with abnormal liver tests, was significantly higher than that of group $B$, the patients with normal liver tests $(\mathrm{p}<0.001)$, with average BMIs being $26.84 \pm 4.99 \mathrm{~kg} / \mathrm{m}^{2}$ for Group A and $25.63 \pm 5.02 \mathrm{~kg} / \mathrm{m}^{2}$ for group $\mathrm{B}$. The frequency of persons with normal or underweight BMI in Group B was significantly higher, at $47.7 \%$, than that of Group A, at $2.7 \%(\mathrm{p}<0.001)$. The frequency of overweight and obese persons was significantly higher in Group A than in Group B ( $\mathrm{p}=0.007$, $\mathrm{p}<0.001$ accordingly) (Figure 2, Table 1). We can conclude that overweight or obesity of all grades is a significant risk factor for pathological processes in the liver.

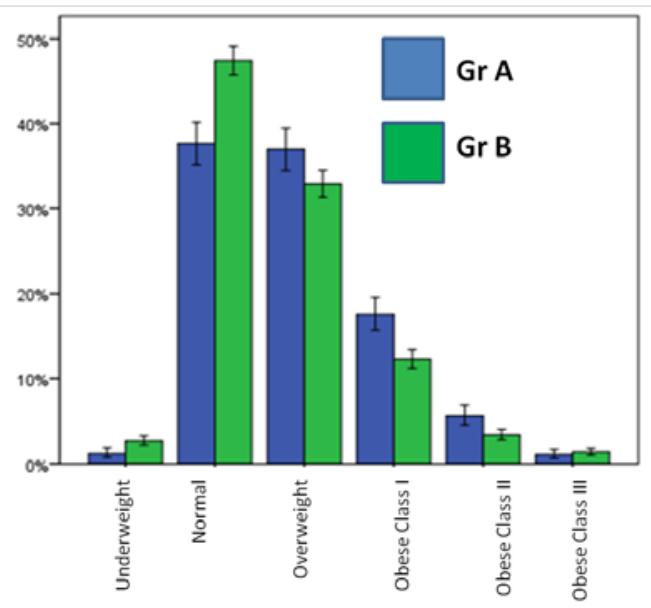

Figure 2. Comparison of the groups according BMI (95\%)

Table 1. Comparison of Groups A and B by average BMI (95\%)

\begin{tabular}{|c|c|c|c|}
\hline BMI & $\begin{array}{c}\text { Group A } \\
(\mathrm{n}=1461)\end{array}$ & $\begin{array}{c}\text { Group B } \\
(\mathrm{n}=3307)\end{array}$ & $\mathrm{p}^{*}$ \\
\hline Average \pm SD & $26.84 \pm 4.99$ & $25.63 \pm 5.02$ & $<0.001$ \\
\hline Underweight & $17(1.2 \%)$ & $88(2.7 \%)$ & $<0.001$ \\
\hline Normal & $547(37.6 \%)$ & $1562(47.4 \%)$ & $<0.001$ \\
\hline Overweight & $537(37 \%)$ & $1084(32.9 \%)$ & 0.007 \\
\hline Obese Class I & $255(17.5 \%)$ & $405(12.3 \%)$ & $<0.001$ \\
\hline Obese Class II & $82(5.6 \%)$ & $111(3.4 \%)$ & 0.004 \\
\hline Obese Class III & $15(1 \%)$ & $44(1.3 \%)$ & 0.477 \\
\hline
\end{tabular}

In our study we also looked at possible relationships between BMI and professional occupation. We found that the groups with the highest average BMI are disabled people, retired people and military personnel - 28.04 $\mathrm{kg} / \mathrm{m}^{2}, 27.93 \mathrm{~kg} / \mathrm{m}^{2}$ and $26.68 \mathrm{~kg} / \mathrm{m}^{2}$ respectively.

\subsection{Laboratory Data}

Significant Liver tests abnormalities were detected more frequently in persons with elevated blood glucose, cholesterol and triglyceride levels $(p<0.001)$. These factors were previously investigated in different international studies and are independent significant risk factors for NAFLD $[1,4,7]$.

\subsection{Smoking}

Smoking prevalence varies greatly from country to country, ranging from $4 \%$ in Libya to $54 \%$ in Nauru. The most "smoking” countries are Guinea, Namibia, Kenia, Bosnia and Herzegovina, Mongolia, Yemen, Turkey, and Romania. Russia is 33rd place in the world ranking.

Only $8 \%$ of the participants surveyed in our study were smokers. There was no difference in smoking rates between Groups A and B: Group A had 8.7\% smokers, group B had $7.3 \%(p=0.101)$. This figure is much lower than data published by WHO in 2011 that reports that $37 \%$ of Russians smoke. We assume this difference is due to the active population recruited for the study (more than $50 \%$ of participants were female) and the positive results of smoking cessation campaigns taking place in Russia since 2010 .

\subsection{Alcohol}

The CAGE and AUDIT questionnaires revealed that a huge number of participants (74.64\% of all participants) should reduce the quantity of alcohol they consume, and that $9.8 \%$ abuse alcohol or are alcohol dependent. The percentage of such persons in Group A was significantly higher than that in Group B, those with suspected "normal” livers. Our data match official Russian statistics published by WHO in 2010 that report that $8.9 \%$ of Russians over 12 years of age abuse alcohol.

\subsection{Anti-HCV and HBsAg Prevalence}

After screening 5,000 people we found that $6.7 \%$ $(n=322)$ were anti-HCV positive, a finding that was reported in 2011 at the International Epidemiological Symposium [15]. $1.9 \%$ of participants $(n=91)$ were HBsAg positive. We analyzed ALT and AST elevation frequency in these persons and could roughly conclude that they have virus replication [10,12]. This represents $50.9 \%$ of anti-HCV positive and $14.3 \%$ of $\mathrm{HBsAg}$ positive participants in our study. These participants need further investigation and antiviral treatment.

We compared anti-HCV and HBsAg prevalence between health care workers and those with other occupations and didn't find any difference: $6.5 \%$ of health care workers are anti-HCV positive compared with $6.9 \%$ of people from other occupations $(\mathrm{p}=0.841)$. HBsAg prevalence is $0.7 \%$ among health care workers, and $1.9 \%$ among other occupations ( $p=0.523$ ). This is probably due to lack of differentiation of health care workers into groups with high and low risk of infection. On the other 
hand, it shows that professional groups at risk consist not only of providers with direct blood contact (surgeons, emergency room physicians, surgical and triage nurses, etc.), but also providers who rarely do invasive procedures and don't have proper safety training.

\section{Conclusions}

According to our study about $30.6 \% \quad(n=1461)$ of Moscow residents have elevated liver function tests (ALT, AST, GGT, AP, total bilirubin, anti-HCV, HBsAg) with a significant male predominance $-49.7 \%$ of all males and $25.5 \%$ of all females $(\mathrm{p}<0.001)$.

Preliminary diagnoses were assigned based on established guidelines [11]. Using these diagnoses, we concluded that the prevalence of liver disease in our study was $(n=4768)$ : NAFLD $7.4 \% \quad(n=352)$, ALD $6.9 \%$ $(n=264)$, hepatitis C 6.7\% $(n=322)$, hepatitis B $1.9 \%$ $(n=91)$, DILI $0.82 \%(n=39)$, cholestasis $0.69 \% \quad(n=33)$, AIH $0.78 \%(n=37)$, other 5.4\% $(n=323)$ (Figure 3).

The main risk factors for abnormal liver tests as determined by multivariable analysis were young age (3059 years, $\mathrm{p}<0.001$ ), alcohol abuse (AUDIT score $>16$, $\mathrm{p}<0.04)$, and hypercholesterolemia $(\mathrm{p}=0.016)$. Univariable analyses revealed additional risk factors to be male sex $(\mathrm{p}<0.001), \quad$ BMI $>25 \mathrm{~kg} / \mathrm{m}^{2} \quad(\mathrm{p}<0.001)$, hyperglycemia $(\mathrm{p}<0.001)$, and hypertriglyceridemia $(\mathrm{p}<0.001)$.

About $80.3 \%$ of patients with abnormal liver tests required etiotropic treatment.

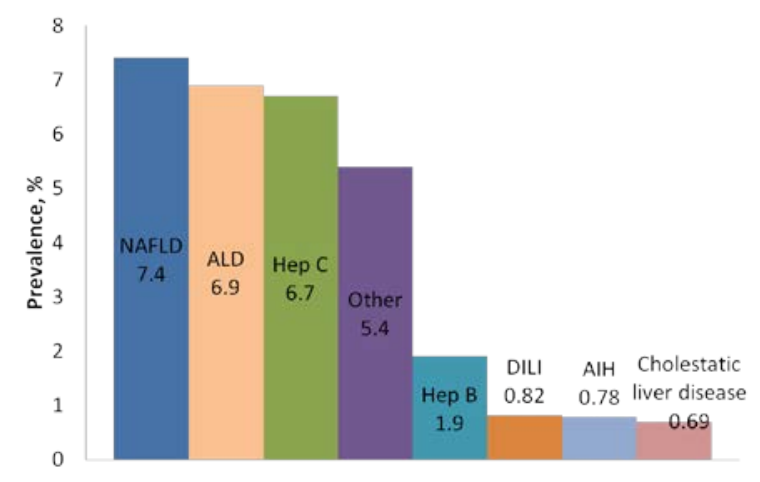

Figure 3. Liver disease prevalence in Russia’s largest city $(n=4,768)$

\section{Acknowledgment}

We thank T.Usipov, D. Colleen for their technical assistance. The authors declared that they do not have anything to disclose regarding funding or conflict of interest with respect to this manuscript.

\section{References}

[1] Bedogni G, Miglioli L, Masutti F, Castiglione A, Croce LS, Tiribelli C, et al. "Incidence and natural course of fatty liver in the general population: the Dionysos study”, Hepatology, 46: 13871391. 2007.

[2] Bell BP, Manos MM, Zaman A. “The epidemiology of newly diagnosed chronic liver disease in gastroenterology practices in the United States: results from population-based surveillance”. Am J Gastroenterol, 103(11): 2727-36; Nov. 2008.

[3] Bueverov A.O., Gotie S.V., Eramishancev A.K., Zhdanov K.V., Ivashkin V.T., Lobzin U.V., Maevskaya M.V., Nikitin I.G., Chzhao A.V. Diffuse liver diseases diagnosis and treatment, Moscow, 2003, 68.

[4] Chalasani Naga, Younossi Zobair et al. "The Diagnosis and Management of Non-Alcoholic Fatty Liver Disease: Practice Guideline by the American Association for the Study of Liver Diseases", American College of Gastroenterology, and the American Gastroenterological Association. 2012.

[5] Ghany M. G., Strader D.B., Thomas D.L., Seeff L.B. Diagnosis, Management, and Treatment of Hepatitis C: an update. AASLD Practice Guideline update, 2009.

[6] Ivashkin V.T., Maevskaya M.V. Alcohol and virus induced liver diseases, Moscow, 2007, 156.

[7] Imhof A, Kratzer W, Boehm B, Meitinger K, Trischler G, Steinbach G, et al. "Prevalence of nonalcoholic fatty liver and characteristics in overweight adolescents in the general population”, Eur J Epidemiol. 22:889-897. 2007.

[8] Lok Anna S. F., McMahon B. J. Chronic Hepatitis B. Update AASLD Practice Guideline update, 2009.

[9] Maevskaya M.V., Ivashkin V.T., German E.N. "How to investagate patients with abnormal liver tests and absence of symptoms”, RGGGC, V.23, №4, 45-68, 2013.

[10] Maevskaya M.V. Chronic liver diseases induced by alcohol and hepatitis B and C viruses, thesis for a Doctor's degree, Moscow, 2006.

[11] Maevskaya M.V., Ivashkin V.T., Method to screen liver diseases and system for it’s realization, patent № 2523661 (Russia); 22.08.2013, 84.

[12] Manns Michael P., Czaja Albert J., Gorham James D. et al. Diagnosis and Management of Autoimmune Hepatitis, AASLD Practice guidelines. 2010.

[13] Martin Blachier, Henri Leleu, Markus Peck-Radosavljevic, Dominique-Charles Valla and Françoise Roudot-Thoraval The Burden of liver disease in Europe. A review of available epidemiological data. 2013.

[14] O’Shea Robert S., Dasarathy Srinivasan, Arthur J. McCullough, and the Practice Guideline Committee of the American Association for the Study of Liver Diseases and the Practice Parameters Committee of the American College of Gastroenterology, Alcoholic Liver Disease, Hepatology. 2010.

[15] Shahgildyan I.V., Ershova O.N., Michailov M.I. et al. "Modern acute and chronic hepatitis C statistics in Russia”, International epidemiological symposium, Brest, 184-186, 2011. 